\title{
Effects of Component Properties on the Accuracy of a Joint-Dominated Deployable Mast
}

\author{
O. R. Stohlman*and S. Pellegrino ${ }^{\dagger}$ \\ California Institute of Technology, Pasadena, CA 91125
}

\begin{abstract}
Jointed-dominated deployable masts are used in space applications, including telescopes, where there is a demand for a long, slender structure that can be packed into a conventional launch shroud. Depending on the application, there may be stringent demands on the stiffness and shape accuracy of the mast. This paper presents a parametric study of the significance of a number of mast part properties for a precision deployable mast's performance under quasi-static shear load. The mast's cable preload, latching system behavior, and joint friction are explored as candidates for parts requiring detailed characterization for accurate mast performance prediction.
\end{abstract}

\section{Introduction}

Deployable masts are a class of structures that can be stowed into a small volume and expanded into long, slender, and stable booms: they can typically be packed to under $5 \%$ of their deployed length. ${ }^{1}$ There are a variety of different designs that have been addressed in previous studies, including early work on a folding articulated truss ${ }^{2}$ for a tethered satellite experiment, a number of versions of the Astromast, ${ }^{3}$ which has been used to deploy the Mars Odyssey gamma ray spectrometer boom, and the recent ST8 SAILMAST ${ }^{4}$ experiment that characterized the performance of a coilable boom, appropriate for gossamer structures.

This paper is concerned with precision deployable masts that can provide a repeatability of the deployed tip position on the order of a few millimeters over a length of tens of meters.

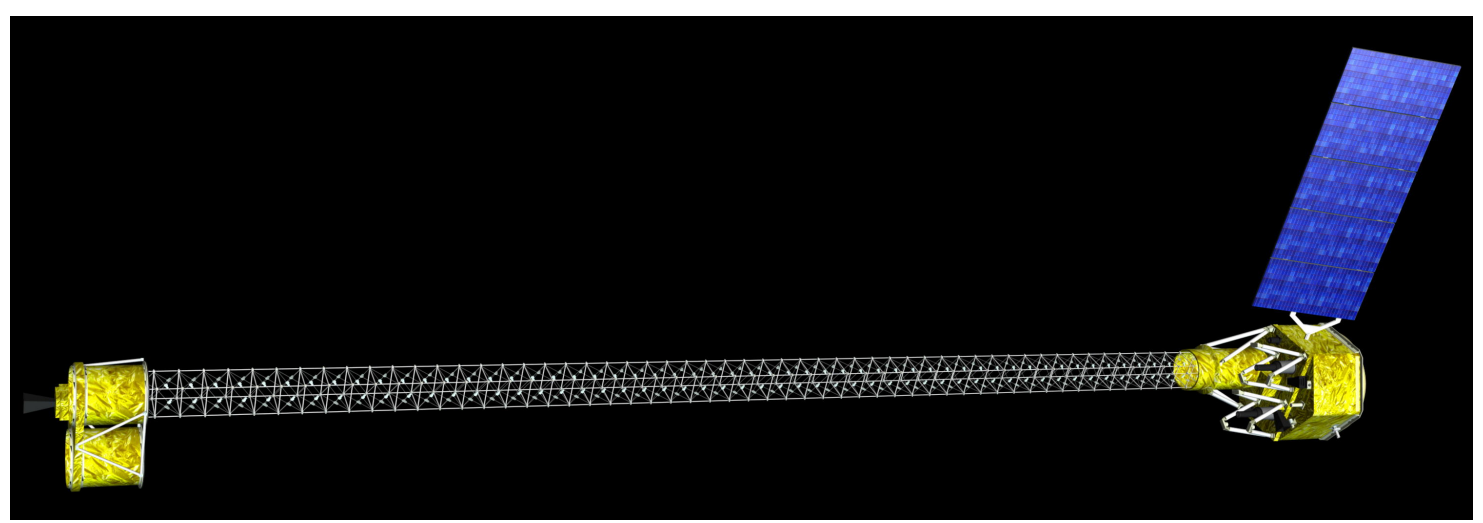

Figure 1. Artist's image of NuSTAR, with the optics at left and detectors/spacecraft bus at right, shows the deployable mast that NuSTAR will use to maintain its focal length of approximately $10 \mathrm{~m}^{\text {(image from NASA }}$ ). $^{2}$

A recent high-precision application for deployable masts is as part of X-ray telescopes, where the grazing optics must be kept a long distance away from the focal plane sensors. The first space observatory that will use a deployable mast is the upcoming Nuclear Spectroscopic Telescope Array (NuSTAR) ${ }^{5}$ X-ray observatory; it

*PhD Candidate, Graduate Aerospace Laboratories, 1200 East California Boulevard, Mail Code 205-45, Member AIAA. e-mail: olive@caltech.edu

$\dagger$ Joyce and Kent Kresa Professor of Aeronautics and Professor of Civil Engineering, Graduate Aerospace Laboratories, 1200 East California Boulevard, Mail Code 301-46, Fellow AIAA. e-mail: sergiop@caltech.edu 
will use a $10 \mathrm{~m}$ deployable mast system. A small variability in the deployed length of this mast does not affect the performance of the telescope, but the tolerance for lateral misalignment is much tighter. Because the lateral precision, repeatability and sensitivity to gravity loading during ground testing are not sufficiently known for existing masts, NuSTAR will incorporate an adjustment mechanism in the last section of the mast, to enable one-time on-orbit alignment. ${ }^{6}$ The particular deployable mast that this study will focus on is produced by ATK Aerospace Systems in Goleta, CA and is called ADAM. This mast is of special interest because it has been used as a precision structure in multiple space missions, including the $60-\mathrm{m}$ baseline Shuttle Radar Topography Mission. ${ }^{7 ; 8}$ It was also the subject of a study on thermal snap conducted from the space shuttle on STS- $85,{ }^{9}$ and is the structure chosen for NuSTAR.

There are many sources of variation in a mast's tip position. Dynamic effects are well understood and thermal distortion effects drive a great deal of material selection and general design, ${ }^{10}$ they are present in every type of mast and of course also in the ADAM mast. Secondary to thermal distortions are mechanical sources of static position error. Aside from catastrophic mechanical failures, the mechanical sources of change in the tip positions are history-dependent material properties and history-dependent interfaces. As with thermal distortions, all masts are similarly subject to material plasticity, strain hardening, and cyclic damage.

In addition to the above listed effects, joint-dominated masts are especially subject to friction and other interface effects. It has been demonstrated by Warren et al. ${ }^{11}$ that, under certain circumstances, appropriate transient disturbances to a structure can encourage a series of microdynamic stick-slip events that return a structure to a reliable position. This sort of facilitated relaxation may ameliorate some complications of friction, but adds another subsystem to the spacecraft. Gravity effects during ground testing can also add a further source of error ${ }^{12}$ in predicting on-orbit behavior of a mast.

Most deployable masts are based on modular designs. As such, they contain many nominally identical parts, and those parts will have a certain spread in their properties. With many properties this spread is not consequential; the effect is often very small, or averaged out over many bays, or revealed and accounted for after ground testing. It is not obvious whether this is true of every stochastic property of mast parts, and this paper will attempt to identify the impact of a few selected properties with known distributions. Hence, two particular effects, joint-driven hysteresis and the impact of stochastic part properties, are the subjects of this paper. They are concerns characteristic of modular, joint-dominated structures, and understanding the magnitude of their impact on mast performance is the broad goal of this study.

This paper will focus on parametric studies that reveal the importance of certain stochastic and frictional properties of an ADAM mast through finite element modeling. The remainder of the paper is outlined as follows. First, an explanation of our present approach to the problem is presented. In section II.A, an overview of some measured properties of a sample mast is given, and the approach in concluded in section II.B with a description of the modeling methods. The parametric study is presented against a measurement of a short two-bay mast loaded in shear, as described in section III.A. The results of parametric studies on the longeron joints (section III.B), diagonal cables (section III.C), and latching system (section III.D) are discussed in the concluding section IV.

\section{Approach}

The approach of this study is rooted in experiment and numerical modeling. The ultimate product is a framework for modeling a mast with stochastic properties, friction, preloaded cables, and nonlinear parts. The study model is valid for large displacements, including displacements that engage the nonlinear effects of the deployment locking mechanism.

Two types of experiments are needed to build a numerical model of the mast: part characterization and mast characterization. The part characterization focuses on hysteretic and stochastic properties, which are especially important for the goals of this study. Mast characterization refers to measurements of the behavior of one or more bays of an assembled mast. These measurements are essential to the model validation.

The experiments were performed on the 6-bay ADAM mast shown in figure 2, whose bays are all nominally identical. Each bay is bounded by rigidly-jointed square batten frames, with hinged longerons along the edges, and each face is braced by a cable assembly consisting of three cables, two shorter and one longer. The shorter cables connect the corner joints of a batten to a latching mechanism, consisting of a pulley and a set of jaws, that is located at the center of the face when the mast is fully deployed. The longer cable passes over a pulley contained within the latching mechanism and is connected to the corner joints of the 
other batten at the two ends. When the bay is fully deployed a bead attached to the longer cable is captured by the jaws, then the bay face is triangulated and the structure is rigid. When the bead is outside the latch, the longer cable can move over the pulley in a way that allows the bay to be stowed through the motion shown in figure 2 .

The main properties of the ADAM mast that has been investigated are summarized in Table 1.
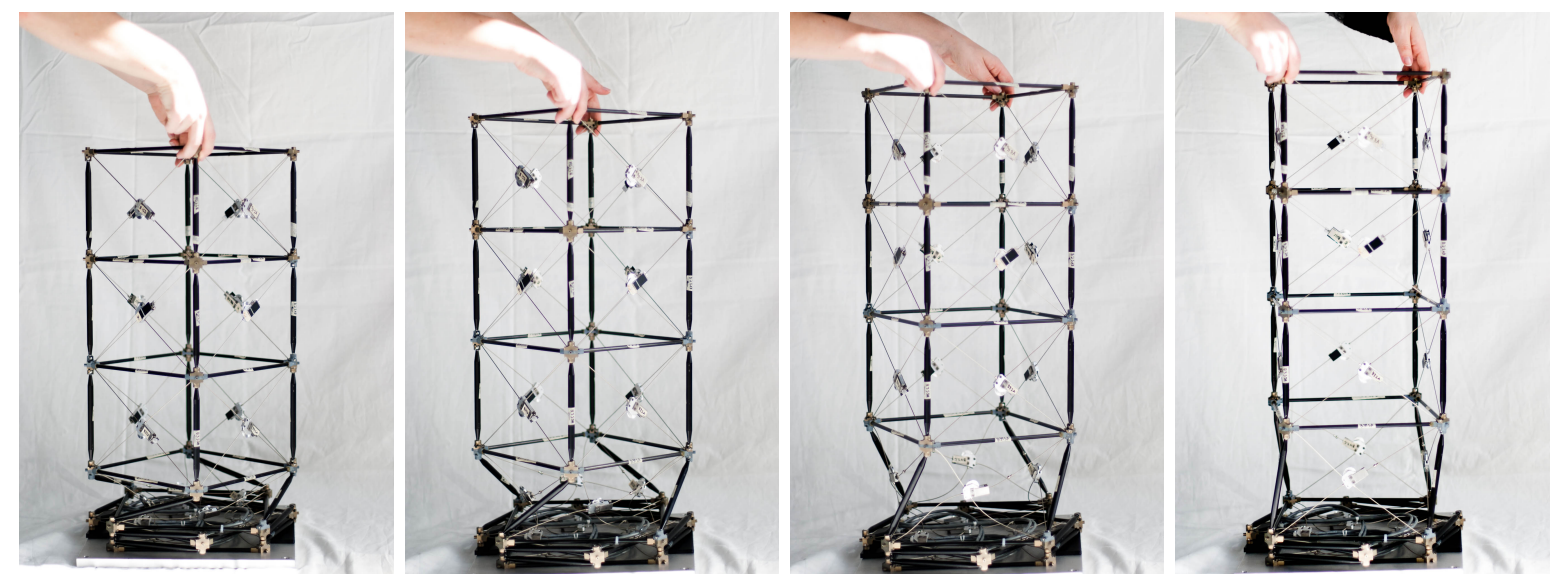

Figure 2. ADAM mast used in this study, shown with three fully deployed bays and a single partially deployed bay.

Table 1. Properties of model mast

\begin{tabular}{ll}
\hline \hline Bay height & $170 \mathrm{~mm}$ \\
Bay width & $230 \mathrm{~mm}$ \\
Ball-end joint diameter & $8 \mathrm{~mm}$ \\
Cable stiffness & $52 \mathrm{kN}$ \\
\hline \hline
\end{tabular}

\section{II.A. Experimental properties}

In order to determine appropriate ranges for the part properties and the manufacturing tolerances that should be considered in the model, a variety of measurements were taken from the sample mast. The measurements were directed towards (i) properties that affect friction and (ii) properties with measurable variability. In the former category are the moving parts: the longeron ball-end joints and the latching mechanisms. The latter category again includes measurements of the ball-end joint friction and the latching mechanism behavior, and also addresses the preload in the diagonal cables of the deployed structure.

The longeron ball-end friction was measured by fastening a longeron and its end joints in a torsion testing machine, by applying an axial compression load and then recording the twisting moment as a function of the applied torsional rotation. This method produced estimates of the friction coefficient in the range $\mu=0.03$ to 0.25 for different longerons.

The latching mechanisms were characterized by measuring the relationship between the difference in tension across the cable on either side of the bead, $\Delta T$, (applied by the latch jaws) and the position of the bead, $x$. These measurements were taken with a standard materials testing machine and a typical set of results is shown in figure 3. The regions labeled (a), (b) and (d) in this plot are of interest to study the folding and deployment of the mast, but under normal operating conditions the region labeled (c) is of greatest interest. This region corresponds to the bead being pushed against the backstop by the latch jaws. The details of the region labeled (c) have been assessed in 30 experimental measurements of 12 different latching mechanisms.

Finally, using a vibration method ${ }^{13}$, the cable preload levels in a four-bay length of the sample mast were identified. These values, shown in the histogram in figure 4, ranged from 130 to $330 \mathrm{~N}$, with a mean of 220 $\mathrm{N}$ and standard deviation of $50 \mathrm{~N}$. 

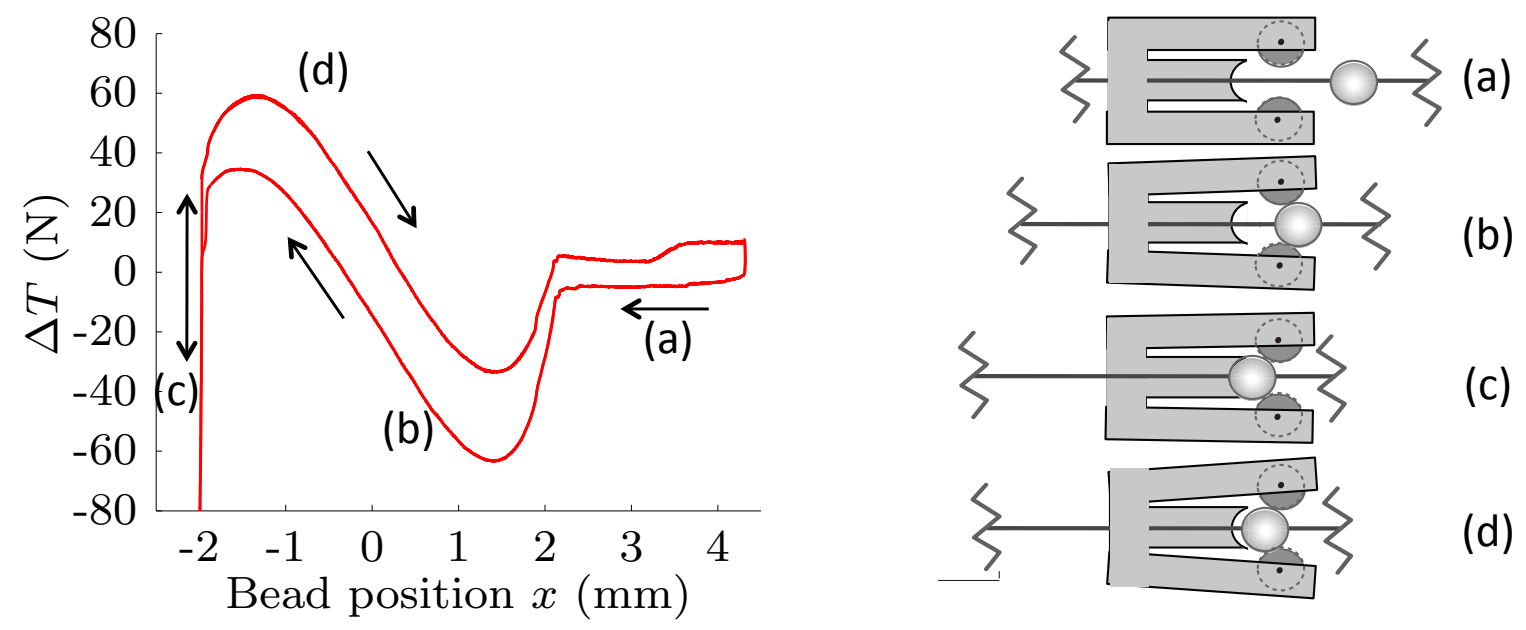

(b)

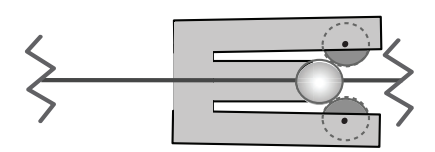

(c)

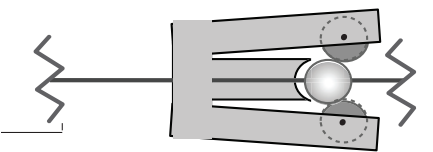

(d)

Figure 3. Experimentally obtained constitutive relationship for a latching element and side views of latching mechanism showing different bead positions.

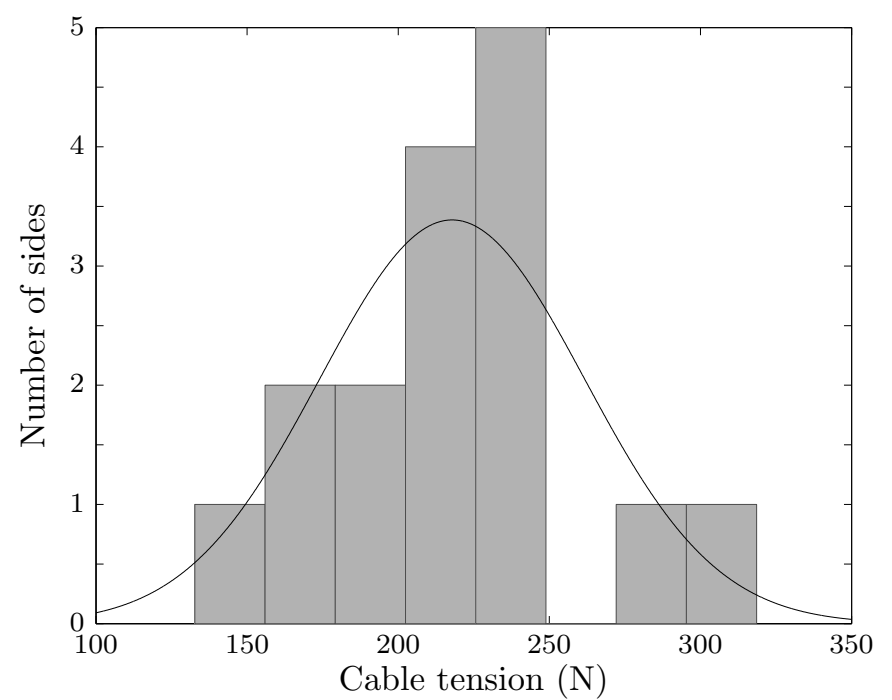

Figure 4. Histogram with normal distribution overlaid of cable preload values for sixteen bay faces of a fully deployed 4-bay mast.

\section{II.B. Finite element model}

A database of experimentally derived part properties was maintained with Matlab, which was also used to generate the input files for the finite element solver Abaqus/Standard. The model simulations were run in Abaqus, which calls a Fortran user subroutine ${ }^{13}$ that computes the behavior of the latch.

The structural elements of the mast were modeled as follows. The longerons were modeled as linear-elastic beams (Abaqus element type B33) with ball joints at either end. The batten frames were also modeled with linear-elastic beam elements connected at the corners by rigid joints of size matching that of the physical model. The cables were modeled as linearly elastic truss elements, with zero stiffness in compression so that they would only able to carry tension loads.

The corner joints of each bay and the bodies of the latch mechanisms were modeled as finite-sized rigid bodies. Unlike the battens, which are firmly glued to the corner joints, the longerons are connected to the corner joints by ball-end joints with significant friction. These were modeled with CONN3D2 elements. Simple Coulomb friction was assumed, with the same friction coefficient for both static and kinetic friction. Based on experimental measurements, the friction coefficients at the joints were assigned randomly with an average 
of 0.25 and a normal distribution with standard deviation 0.125 ; values below 0 were rejected.

The relationship between the axial load $F$ on a longeron and the moment opposing the rotation of its end joints, $M$, is

$$
M \leq \mu r F
$$

in static friction conditions, and

$$
M=\mu r F
$$

when there a relative rotation of the joints occurs. Here $r$ is the radius of the longeron ball-end.

The combination of detailed characterization of the components of the sample mast and the extensive control over its model, as described above, makes it possible to truly see what properties of the mast govern its performance. This approach may help to establish a level of confidence in the behavior of existing masts and to focus future design and manufacturing effort in the areas of greatest impact.

\section{Parametric Studies}

\section{III.A. Reference experiment}

Transient loading cases are the central focus of this modeling problem. One of the important capabilities of the model is predicting what happens to the resting shape of the mast after a load-unload cycle. During a slewing maneuver in space, a mast would see transient loads and be expected to return to a position acceptably close to the initial one after the maneuver. If the mast can end up in a shape that puts it outside the acceptable range, it would be required to limit the rate of attitude correction for the spacecraft.

As a reference case for the parametric studies, this behavior was studied with the quasi-static transient loading experiment pictured in figure 5 . The figure shows a two-bay mast loaded in shear to approximately $\pm 70 \mathrm{~N}$ in the $x$ direction, with the shearing load applied at the center of a plate bolted to the top batten square. The maximum load applied in this test was chosen such as to avoid the spontaneous unlatching and collapse of the mast, which occurred between 80 and $130 \mathrm{~N}$ of shear force, so $70 \mathrm{~N}$ was very near the edge of the tolerable loads for such an experiment. The loading was applied by means of lead shot, which was poured to and from buckets hanging from the load cables shown in figure 5. A typical loading pattern, as recorded by the inline force transducers (Futek model LCM300), is shown in the plot at the top of figure 6 .

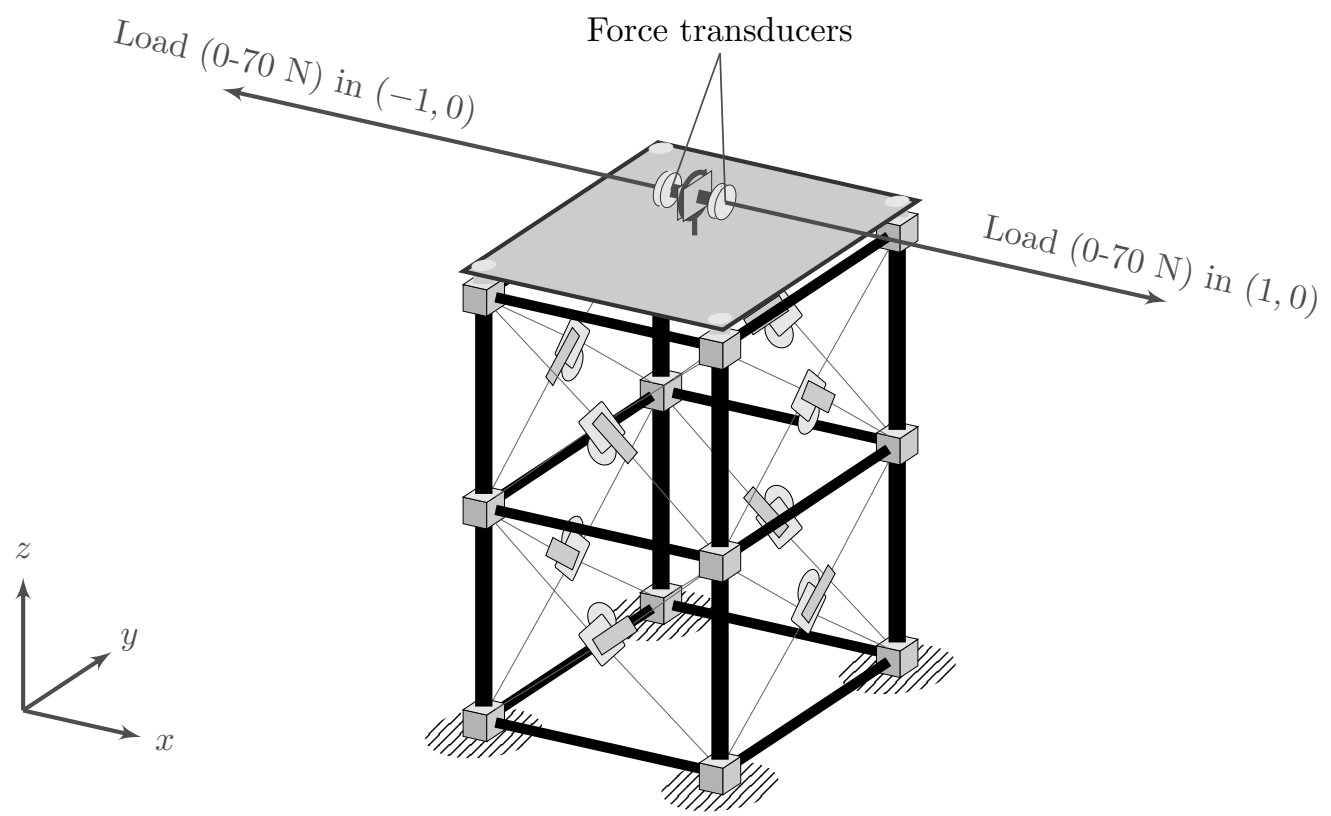

Figure 5. Schematic of shear loading experiment; the $x$-loading cables lie in a plane $57.5 \mathrm{~mm}$ above the center of the top batten square. 

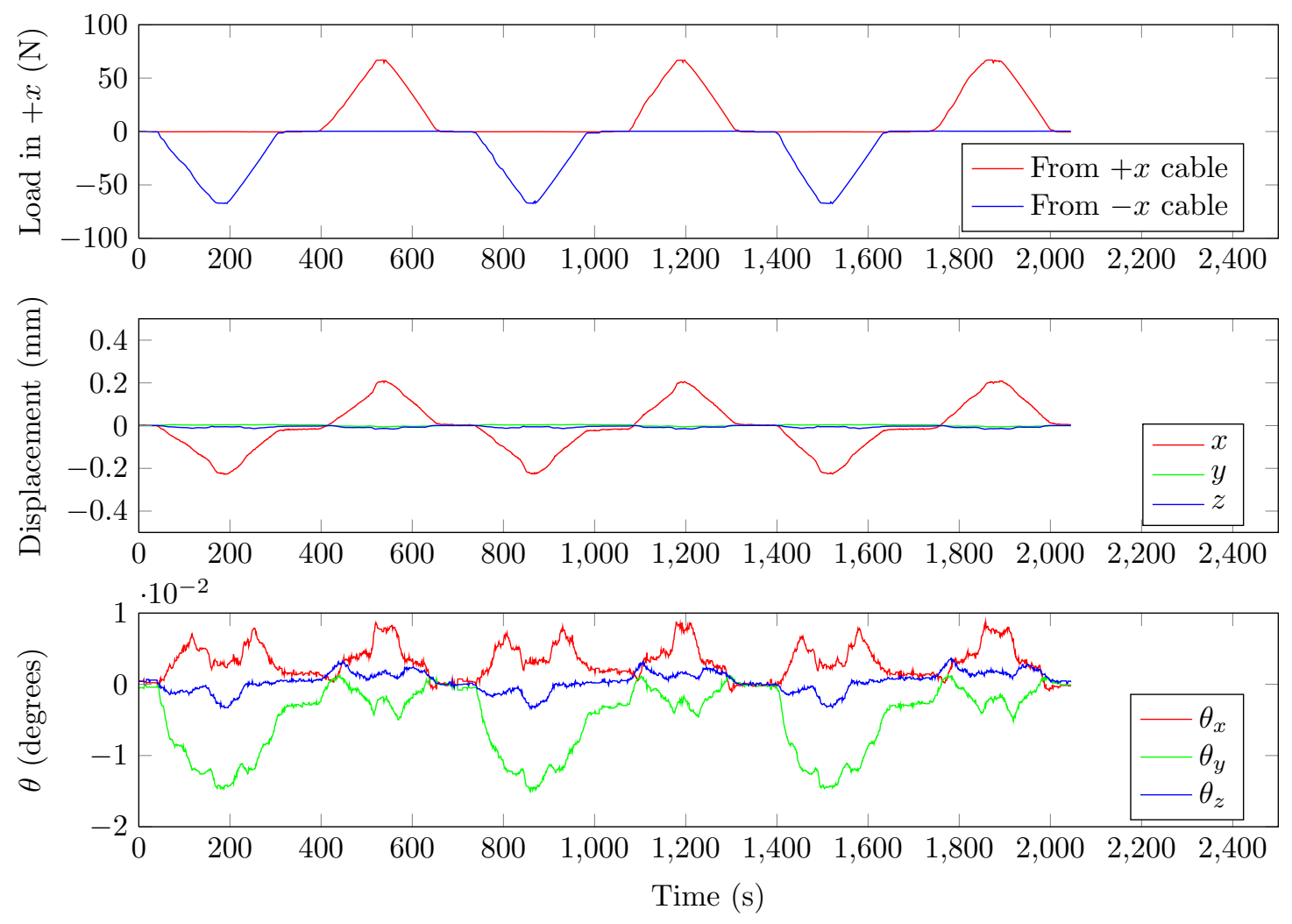

Figure 6. Load and displacement measurements for shear experiment.

The displacement and rotations of the top batten square were measured by a system of six rangefinding lasers (Keyence model LK-G87) and processed to give the six degrees of freedom of the (presumed rigid) top plate and batten square. This laser system is the source of the position and rotation data of figure 6 , and the positions and rotations refer to an imaginary node at the center of the top batten square.

The following simulations all use the boundary conditions of this experiment. That is, the four base corners of the two-bay mast are completely fixed and the top batten square is assumed to move as a rigid body, which is loaded in $\pm x$ at a control node located above its center.

\section{III.B. Longeron ball-end joint friction}

The ball-end joint friction is likely to vary greatly between ground testing and in-orbit use, and even within the orbit. It can be affected to some degree through parameters other than the actual ball-on-socket friction: the joints could be designed with their own pre-load (although they are not in the sample mast) and the cable preload can be increased. It is not the only source of friction; there is a lower level of friction in the latches. It is also not the only parameter with variability in the model, as both the latches' behavior and the cable preloads are assumed to vary from face to face. However, as it will be shown, it is the dominant factor in residual deformation after a loading event.

First, there are two extreme cases in which the longeron joint friction does not produce hysteresis. Intuitively, there is nearly no residual displacement at zero load if there is no friction in the longerons $(\mu=0)$, and none at all if the friction is so high that the joints never slip (e.g., at $\mu=2.5$ ). This result can be seen in figure 7 , which also shows a paddle-shaped feature at high loads that appears when the mast is distorted enough to begin to disengage the reversible latching mechanism. Because the latching mechanisms are not identical to one another and because different latches are engaged depending on the direction of the load, this feature is not isotropic. As the latching behavior is randomly distributed over a range of experimentally obtained constitutive models for the latches, each simulation will show slightly different specific latch-related behaviors. Regardless, no simulations with high longeron ball-end friction show this phenomenon. 

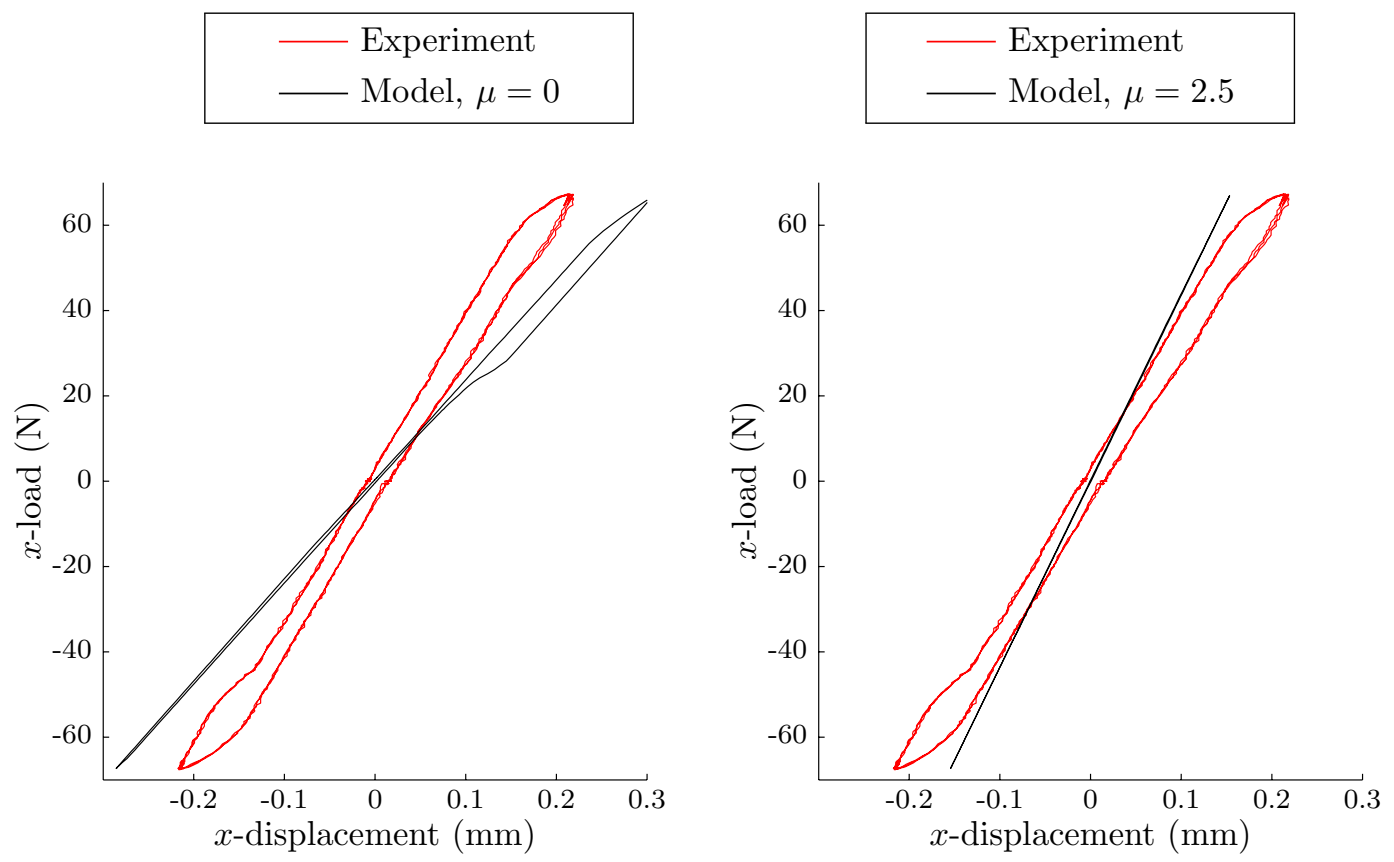

Figure 7. Force-displacement curves of 2-bay mast subject to shear loading, for very high and very low ball-end frictions. Both of these extremes result in virtually no residual displacement at this level of loading, but an asymmetric hysteretic feature is seen in the low-friction case on the left, as in the experiment.

Experimental measurements showed a range of tens of microns in the possible resting positions of the mast, measured by the width of the hysteretic response at zero load. For frictionless ball-end joints, figure 7 does show a small effect, of the order of a few microns, from the friction of the latching mechanisms alone. While this effect is swamped by the ball-end friction on the sample mast, it its nonetheless identifiable in the results from the numerical model.

The precise width of the displacement range for zero load is dependent upon the precise value of the ball-end friction. Because the longerons of the sample mast had unknown lubrication and surface finish properties, a wide range of frictions was considered possible. As shown in figure 8, the value of the friction has a strong effect on the width of the force-displacement curve.

The value of the longeron ball-end friction may thus be concluded to have a measurable effect on position error after a loading event. What is the impact of a distribution of the ball-end friction? The simulations above assumed a distribution of $\mu$ values generated with a standard deviation of $50 \%$ of the friction value (discarding values below zero). Figure 9 shows a number of simulations run with $\mu=0.25$, contrasting simulations with and without a distribution in this value. The spread in behavior is small in either case, but does not appear to be strongly influenced by the single factor of the spread in longeron ball-end friction.

\section{III.C. Cable preload}

Experimental measurements of cable preloads in a deployed mast, in Section II.A had shown a distribution of preload values of $\pm 50 \mathrm{~N}$ around a mean of $T=220 \mathrm{~N}$, which corresponds to a standard deviation of $\pm 100 \mu \mathrm{m}$ in cable length over the length of each of the cables. Therefore, a normally distributed value for this length error was generated for each bay face at random in each simulation, and applied to each of the face's four cable sections.

Figure 10 shows the effect of this distribution of cable lengths. Much as the effect of the ball-end friction distribution, the distribution of cable preload does not appear to cause large variations in overall behavior.

Cable preload has a significant effect on the mast response to this level of shear load. While the level of preload in cables that remain taut would not be expected to have a direct impact on the stiffness of the structure, the preload does increase the structure's stiffness by increasing the load on the ball-end joints, and thus the effect of their stiffness on friction. Further, the relatively large displacements allowed by very low cable preload levels, much like very low values of the longeron ball-end frictions, begin to disengage the 

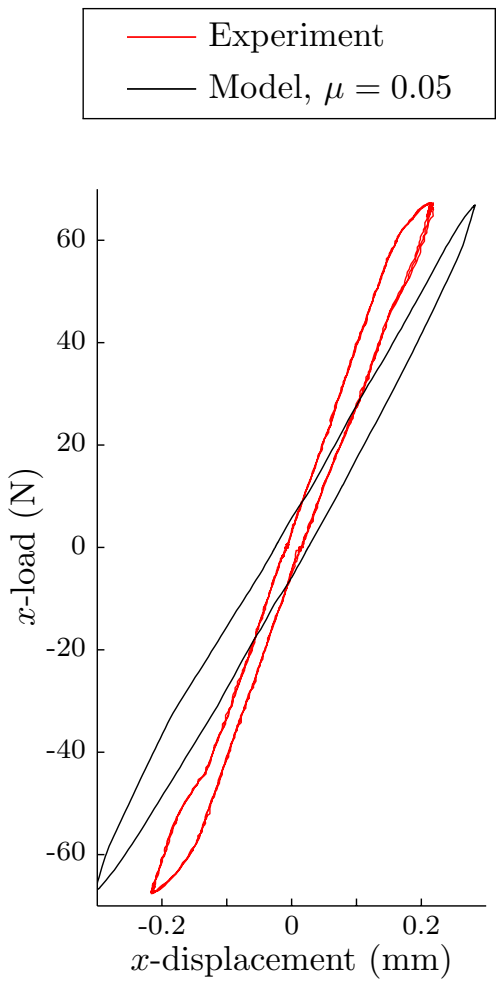
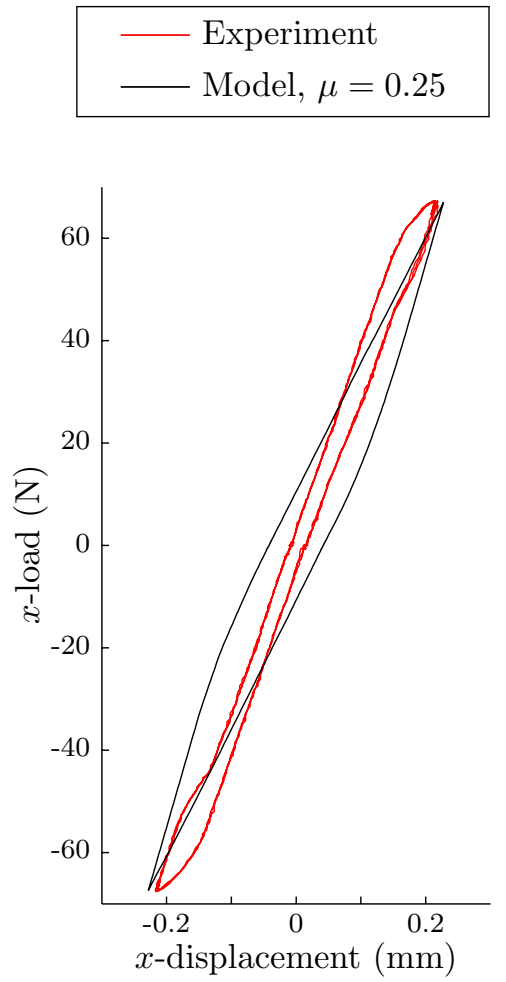
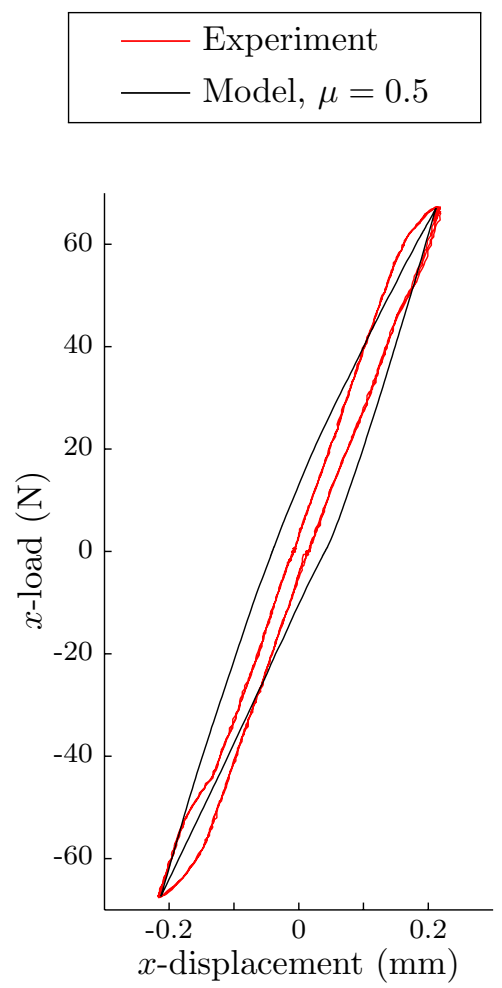

Figure 8. Force-displacement curves of 2-bay mast in shear loading for three moderate values of the ball-end frictions.

$$
\mu=0.25 \pm 0
$$

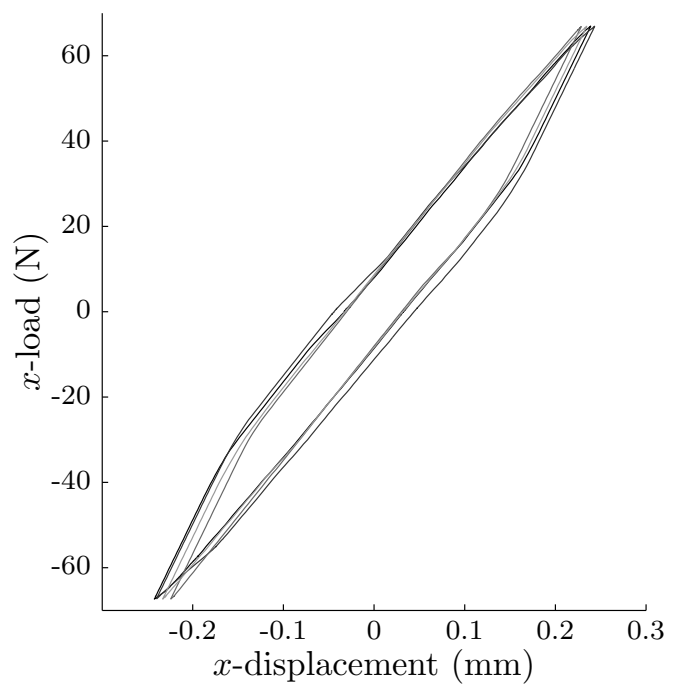

$\mu=0.25 \pm 0.125$

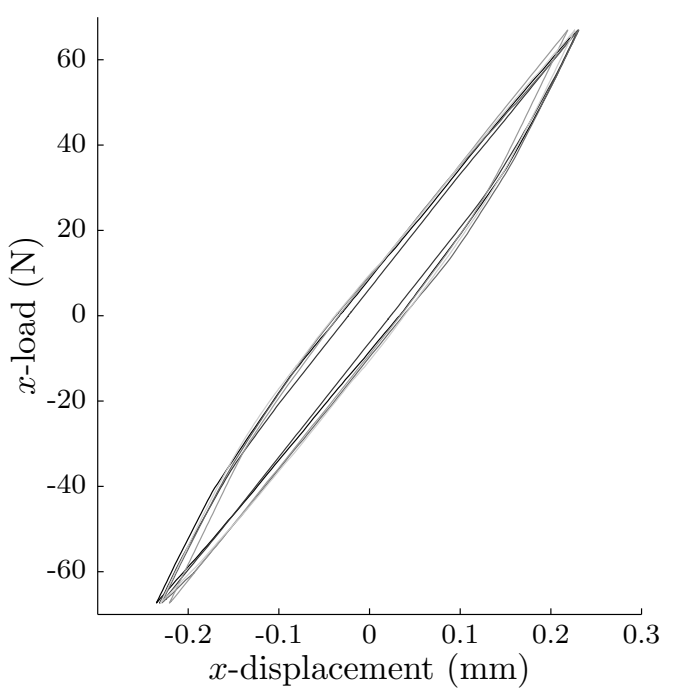

Figure 9. Force-displacement of 2-bay mast with and without distribution in the ball-end friction. Five simulations of each case are shown in different shades of grey. The case with a distribution in the ball-end frictions shows a slightly reduced friction, as the lower-friction end of a given longeron dominates its behavior. 


$$
\sigma_{L_{0}}=0
$$

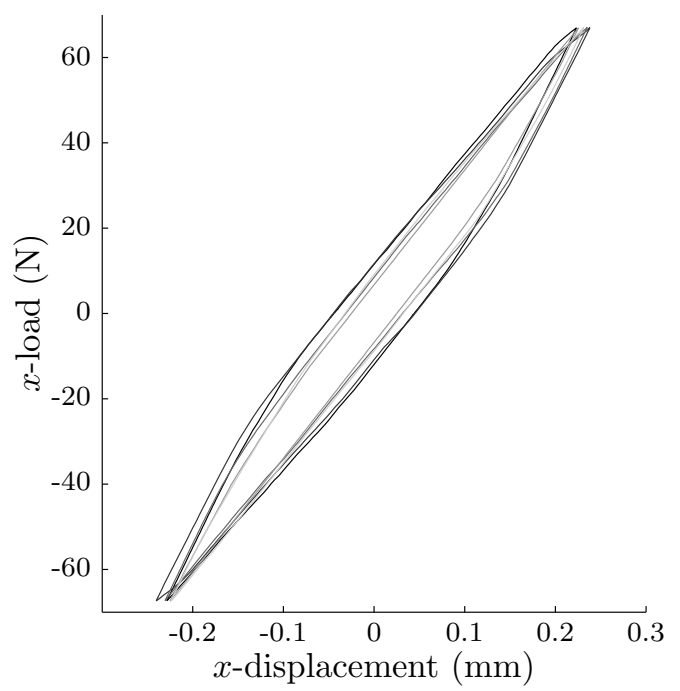

$$
\sigma_{L_{0}}=100 \mu \mathrm{m}
$$

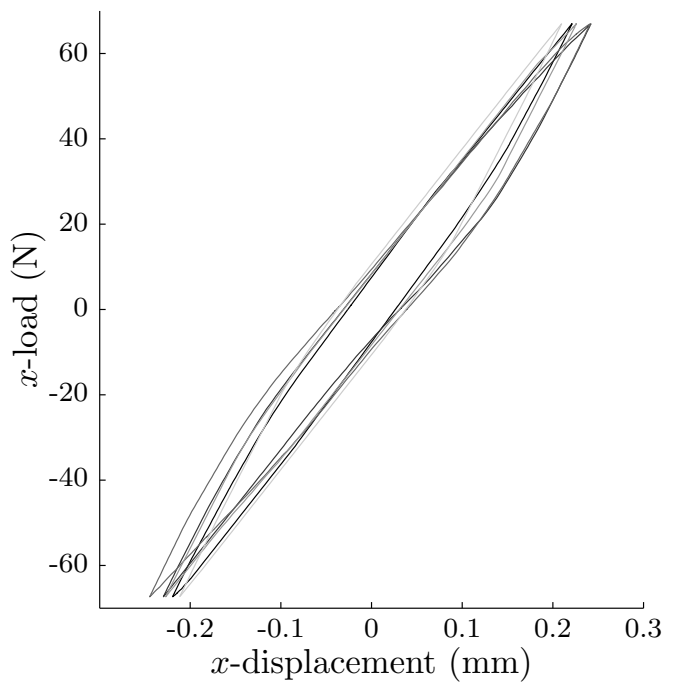

Figure 10. Force-displacement of 2-bay mast with and without distribution in cable preloads. Five simulations of each case are shown in different shades of grey.

reversible latches. This involvement of the latching mechanisms brings out both nonlinearity and anisotropy in the structure's response to shear loading, as seen in figure 11.

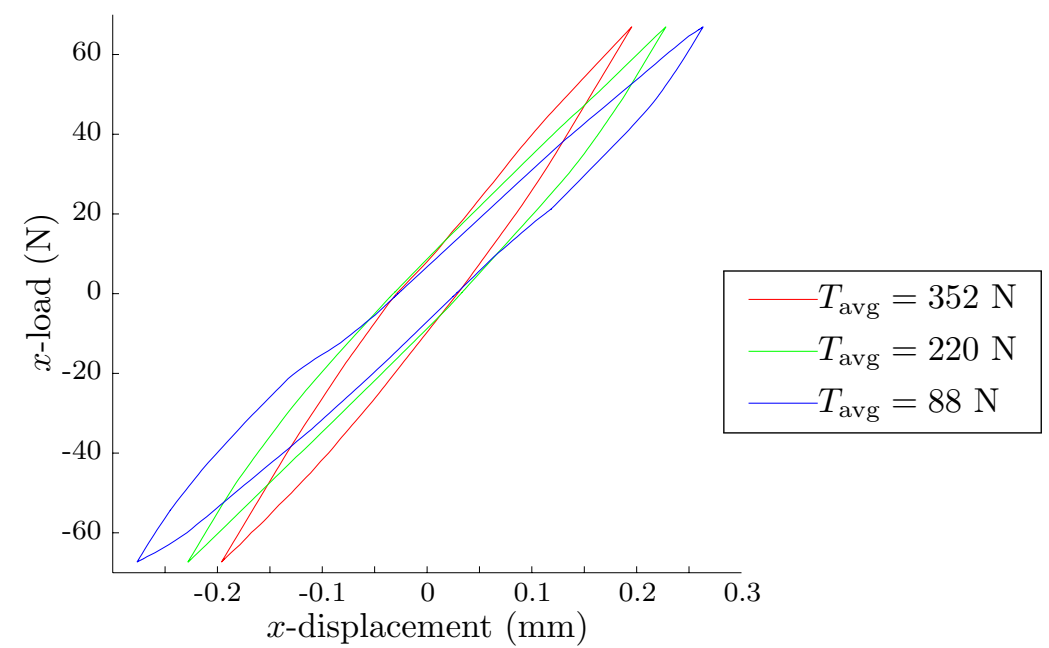

Figure 11. Force-displacement curves of 2-bay mast under shear loading with different levels of cable preload. The differences in load response become more prominent at high loads, and latch involvement is also seen at large displacements, particularly for the $88 \mathrm{~N}$ preload level.

\section{III.D. Measured vs. idealized latch behavior}

The baseline case is the use of a randomly assigned selection of latch constitutive models, each defined by means of a lookup table. It is also possible, and in fact simpler, to use a fit curve to define the constitutive relationship of each latch. The difference in these approaches can be seen, for a representative set of latch data, in figure 12. Each latch in the model was randomly assigned to a set of experimental latch data from the database of 30 experimental measurements of 12 different latches.

Because the area of greatest deviation between these two models is the highly-travelled zone near the backstop, the choice of latch models produces a substantial difference in mast model behavior. The impact on the properties of the mast can be seen in figure 13. Using an idealized analytical model tends to decrease 

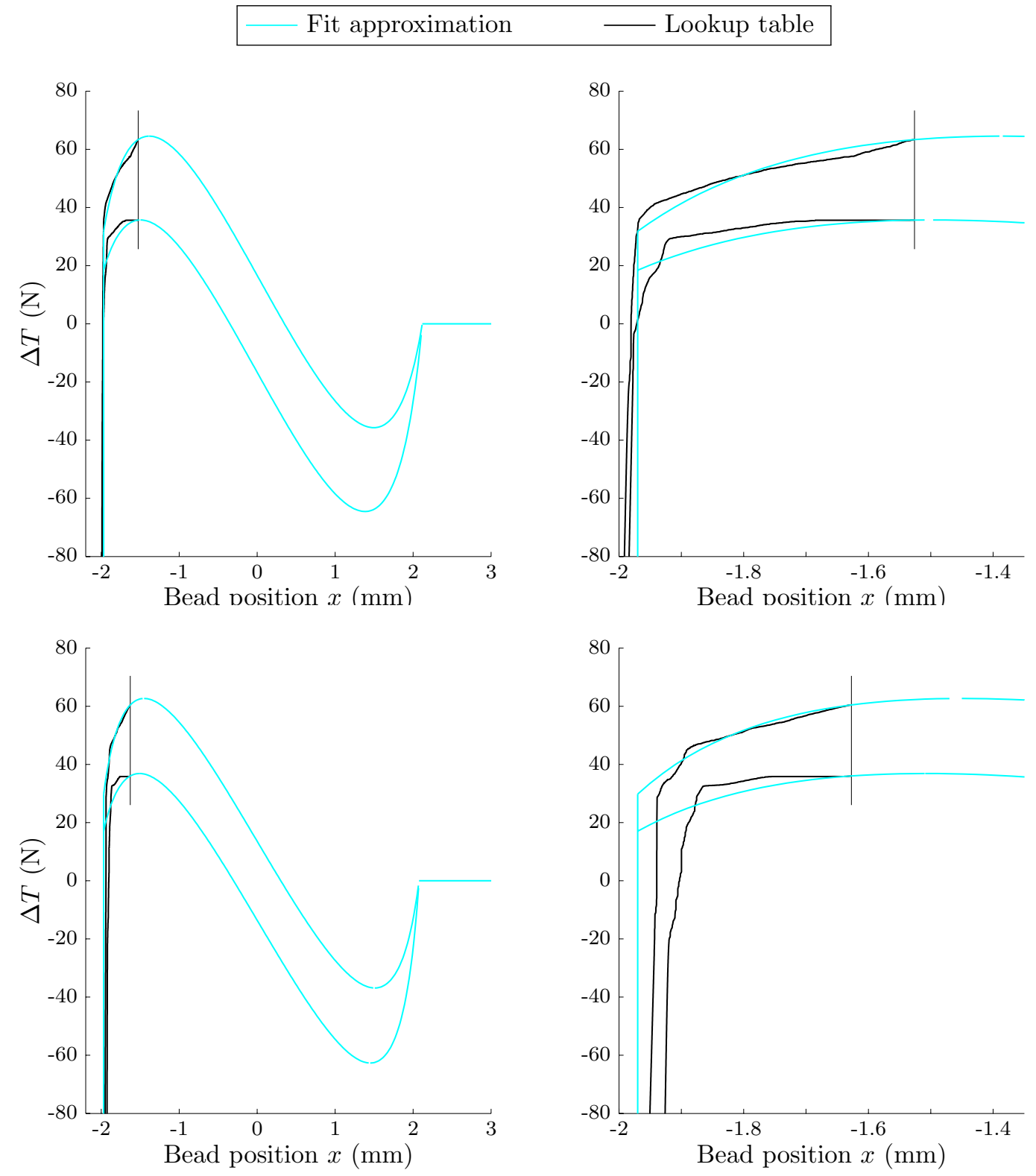

Figure 12. Constitutive relationships for two latching mechanisms: in cyan, profiles based on a fit curve and in black, profiles obtained directly from the experimental data. The figures to the right show an enlargement of the area near the latch backstop. In both versions, a fit analytical approximation to the shape of the experimental data was used to the right of the vertical black line. 
the load at which a characteristic bulge appears in the force-displacement curve. This bulge is caused by the beads of one or more latches leaving the backstop, and indicates that the load is approaching the point of sudden delatching.

With fit curves

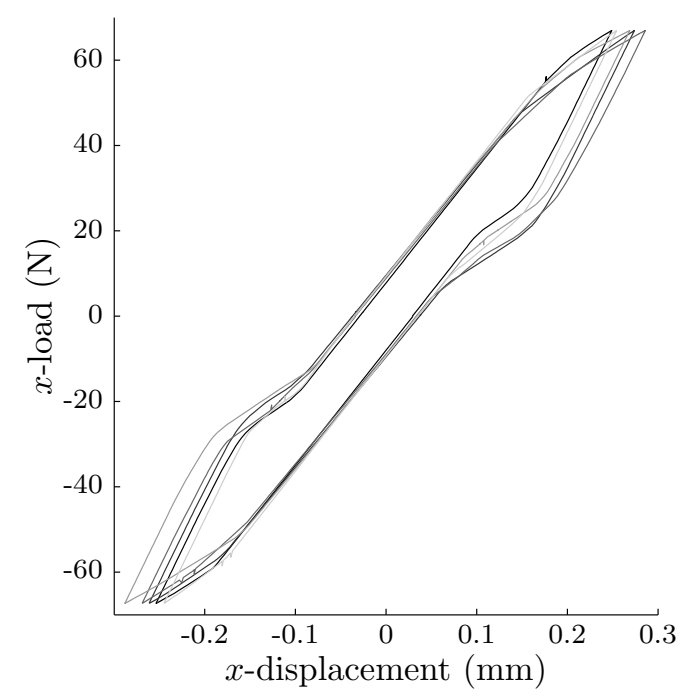

With lookup tables

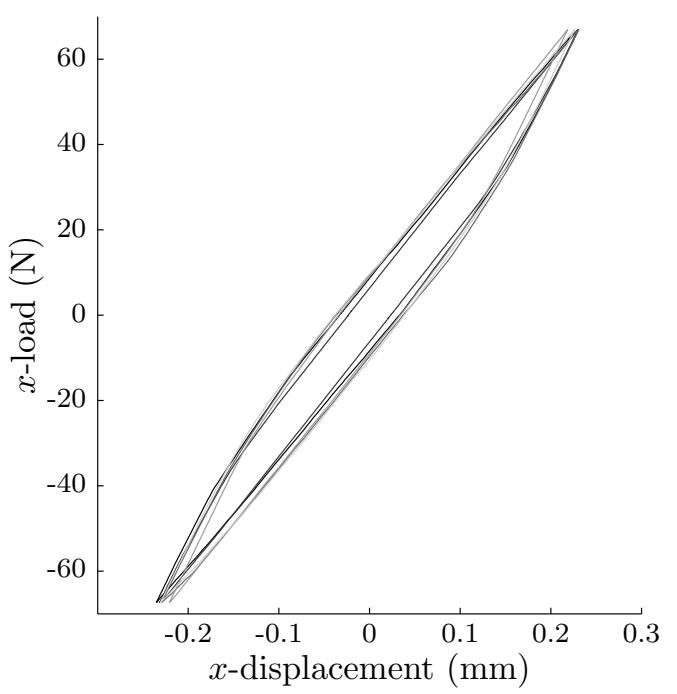

Figure 13. Force-displacement with the two different treatments of the latching mechanism. Five simulations with each type of latch model are shown in varying shades of grey to demonstrate the effects of stochastic properties.

\section{Discussion}

This paper has shown the importance of three properties of a jointed deployable mast: joint friction, cable preload, and the behavior of the latching mechanism. The part variability was most significant in the latches, where differences in behavior produced anisotropy and nonlinearity at higher loads. Cable preload had a distinct impact on the mast stiffness in shear, but preload variability was not shown to have a substantial impact on performance. Finally, the average longeron friction had a large impact on the range of displacement at zero load. This is significant in part because of the difficulty of controlling this friction.

It is noteworthy that cable preload can impact the structure's stiffness through its interactions with the joint friction. The coupling of these parameters emphasizes the importance of including both effects, as together they can have a substantial impact on the stiffness of the structure and its hysteretic behavior.

Anisotropy arising from the latch variability appears at high loads because different load directions engage different latches. This effect would be expected to become more isotropic in longer masts, but the nonlinearity would then be exacerbated by a larger number of latches, all with slightly different constitutive properties, in series. The latches are the most complex moving part in the mast itself, as well as the least stiff. As the locking mechanism for the structure, they are worthy of particular attention because their reaction to high loads determines whether the mast will remain rigid or delatch and fail. In any event where the loading exceeds a few tens of Newtons in shear, a rigid approximation of the latches will not capture the nonlinear stiffness of the mast and may miss coupled distortions of the mast.

The continuation of this work will fit undetermined parameters to the experimental data and pursue parametric studies of longer masts.

\section{References}

1 Able Engineering, "ABLE ADAM articulated boom system," 2004, http://www.aecable.com/Booms/adam.html.

2 Warden, R. M., "Folding, articulated, square truss," 21st Aerospace Mechanisms Symposium, L.B. Johnson Space Center, Houston, Texas, 1987. 
3 Adams, L. R., "The X-beam as a deployable boom for the space station," 22nd Aerospace Mechanisms Symposium, NASA Langley Research Center, Hampton, Virginia, 1988.

4 McEachen, M. E., Trautt, T. A., and Murphy, D. M., "The ST8 SAILMAST validation experiment," 46th AIAA/ASME/ASCE/AHS/ASC Structures, Structural Dynamics, and Materials Conference, Austin, Texas, 2005.

5 Caltech, "Official NuSTAR Homepage," http://www.nustar.caltech.edu/.

6 Harrison, F.A. et al., "The nuclear spectroscopic telescope array (NuSTAR)", SPIE, 7732, $27,2010$.

7 Duren, R. M. and Liebe, C. C., "The SRTM Sub-arcsecond Metrology Camera," Aerospace Conference, IEEE Proceedings, Vol. 4, 2001, pp. 4/2037-4/2046.

8 Umland, J. W., "SRTM Mast Damping Subsystem Design and Failure Investigation," 35th Aerospace Mechanisms Symposium, Ames Research Center, 2001.

9 Levine, M. B., "On-orbit microdynamic behavior of a flexible structure: IPEX II," JPL Technical Report, 1999 .

10 Edeson, R., Aglietti, G. S., and Tatnall, A. R. L., "Conventional stable structures for space optics: The state of the art," Acta Astronautica, Vol. 66, 2010, pp. 13-32.

11 Warren, P. A., Peterson, L. D., and Hinkle, J. D., "Submicron mechanical stability of a prototype deployable space telescope support structure," Journal of Spacecraft and Rockets, Vol. 36, No. 5, 1999, pp. $765-771$.

12 Folkman, S. L., Roswell, E. A., and Ferney, G. D., "Influence of pinned joints on damping and dynamic behavior of a truss," Journal of Guidance, Control, and Dynamics, Vol. 19, No. 6, 1995, pp. 1398-1403.

13 Stohlman, O. R. and Pellegrino, S., "Shape accuracy of a joint-dominated deployable mast," 51st AIAA/ASME/ASCE/AHS/ASC Structures, Structural Dynamics and Materials Conference, 12-15 April 2010, Orlando, FL, AIAA-2010-2605. 